\title{
PHACES syndrome with ectopia cordis and hemihypertrophy
}

\author{
Jad Chokr, MD, FRCR ${ }^{\mathrm{a}}$ (D), Bedros Taslakian, $\mathrm{MD}^{\mathrm{b}}$ (D), Gilbert Maroun, $\mathrm{MD}^{\mathrm{C}}$ (D) and Gagandeep Choudhary, $\mathrm{MD}^{\mathrm{d}}$ (D) \\ ${ }^{a}$ Department of Radiology, Clemenceau Medical Center, Johns Hopkins International, Beirut, Lebanon; bepartment of Radiology, New York \\ University School of Medicine, New York, New York; 'Department of Radiology, American University of Beirut Medical Center, Beirut, \\ Lebanon; 'Department of Radiology, University of Alabama at Birmingham, Birmingham, Alabama
}

\begin{abstract}
PHACES is the acronym describing the phenotypic association of posterior fossa anomalies, facial hemangioma, cardiac and eye anomalies, and sternal defects. To date, more than 300 cases of PHACE(S) have been reported. We present the case of a newborn girl who was born with a variant of the PHACES syndrome. Although the sternal cleft and the small facial hemangioma were evident clinically at birth, magnetic resonance imaging of the brain provided additional information to establish the diagnosis. In addition, the patient manifested later with hemihypertrophy, an association that has not been described previously.
\end{abstract}

KEYWORDS Ectopia cordis; hemihypertrophy; PHACES syndrome; posterior fossa anomalies

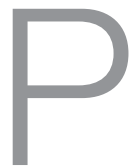

HACES syndrome (the phenotypic association of posterior fossa anomalies, facial hemangioma, cardiac and eye anomalies, and sternal defects) is one of the neurocutaneous syndromes of unknown etiology with no definite chromosomal abnormality identified to date. ${ }^{1}$ Ectopia cordis, an extremely rare malformation, has been described in association with $\operatorname{PHACE}(S)$ syndrome. ${ }^{2}$ It is classified as either complete or partial. In case of complete ectopia cordis, the heart is displaced outside the thoracic cavity without pericardial coverage, as opposed to the partial form where the heart is covered with a thin layer of pericardium. ${ }^{2,3}$ In this case report, we describe an unusual new association of PHACES syndrome with partial ectopia cordis and hemihypertrophy.

\section{CASE REPORT}

A female term infant was born to a 21-year-old gravida 1 , para 0 , aborta 0 , nonsmoking mother after an uncomplicated pregnancy. The parents were nonconsanguineous and did not report any family history of congenital abnormalities. On physical examination, the infant had a complete sternal cleft, with a partially displaced heart outside the thoracic cavity, covered by a thin skin (Figures 1a-1c). Further examination revealed a faint facial hemangioma covering the right cheek (Figures 1a-1c). The remainder of her examination did not show any other abnormality. A plain chest radiograph showed dextrocardia and widely separated clavicles and anterior ribs (Figure 1d).

Magnetic resonance imaging (MRI) of the brain showed a hypoplastic right cerebellar hemisphere, absent inferior vermis, and dilated fourth ventricle, in keeping with the Dandy-Walker variant (Figure 1e). No orbital lesions were present at that time (Figure 1f), and diagnosis of PHACES syndrome was suggested.

Follow-up examination at 1 month showed exophthalmos with a significant increase in the size of the facial hemangioma, which was covering the entire trigeminal nerve distribution (Figures 2a-2c). Echocardiogram showed mesocardia/ mild dextrocardia with slight rightward orientation of the heart apex and aortic coarctation, completing the full spectrum of the syndrome.

Magnetic resonance angiography (MRA) of the intracranial and neck vessels revealed complex arterial anomalies (Figures 2d-2f). Both internal carotid arteries (ICAs) were small in caliber at the level of the neck (Figure 2d), with discontinuity of the right ICA at the skull base and the left ICA in its cavernous portion (Figure 2e). The vertebral arteries

Corresponding author: Gagandeep Choudhary, MD, Department of Radiology, University of Alabama at Birmingham, 619 19th St. S., Birmingham, AL 35249-6835 (e-mail: dr.gagan1984@gmail.com)

Color versions of one or more of the figures in the article can be found online at www.tandfonline.com/ubmc.

Received September 5, 2018; Revised November 4, 2018; Accepted November 5, 2018. 

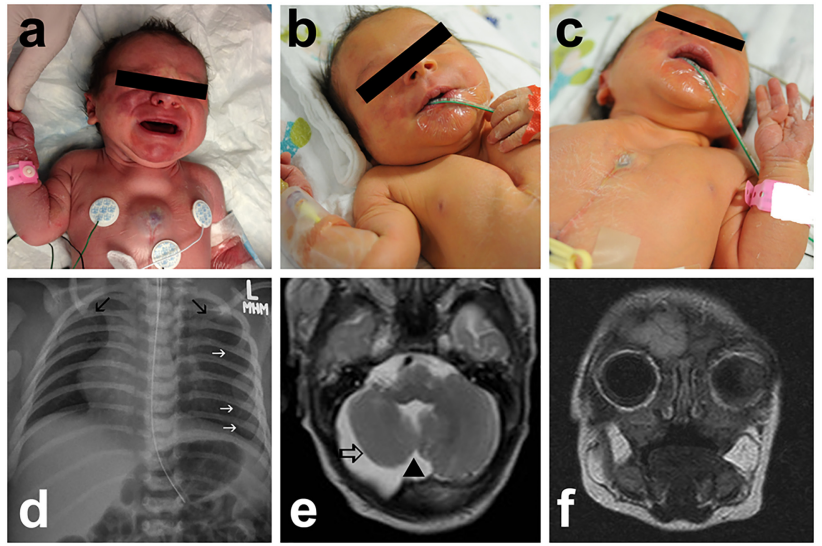

Figure 1. Photograph and imaging at day 1. (a) Faint right facial hemangioma with midline thoracic wall defect and a mid-thorax bulge representing the pulsating heart. $(\mathbf{b}, \mathbf{c})$ Sternal cleft mainly seen during inspiration. (d) Plain chest radiograph showing dextrocardia, widely separated clavicles, and anterior ribs (arrows) suggestive of sternal cleft. (e) Transverse fast spin-echo T2-weighted MRI of the posterior fossa showing a right cerebellar hypoplasia (open arrow), with absence of the inferior part of the vermis (arrowhead), in keeping with Dandy-Walker variant. (f) Coronal T1-weighted image showing normal periorbital fat with no exophthalmos or periorbital lesions.
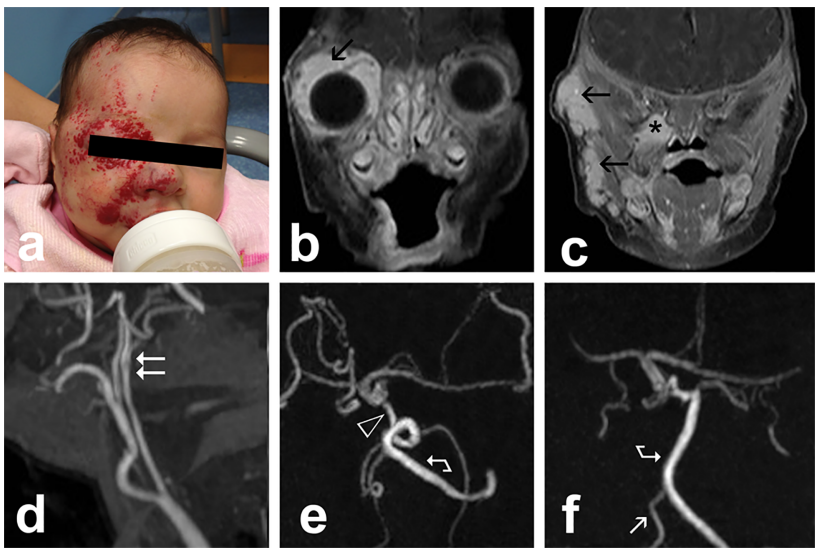

Figure 2. Photograph and imaging at 1 month of age. (a) Large facial hemangioma, covering the whole trigeminal area, with exophthalmos. Coronal T1weighted fat-suppressed spin-echo MRI of the orbits after gadolinium at (b) the level of the orbit and (c) the orbital apex showing an enhancing left orbital and periorbital hemangioma (black arrow), hemangiomas in the right pterygopalatine fossa (asterisk), and the masticator space (black arrows). (d) Right lateral oblique three-dimensional time-of-flight (TOF) magnetic resonance angiography (MRA) of the neck vessels showing small right extracranial internal carotid artery (ICA) ending at the level of the skull base (double white arrows). (e) Axial three-dimensional TOF MRA of the circle of Willis showing prominent basilar artery (white curved arrow) and right posterior communicating artery filling the anterior circulation (open arrowhead). (f) Posterior threedimensional TOF MRA of the circle of Willis showing absent intracranial segments of the right and left ICAs, with prominent posterior circulation. Note the prominent basilar artery (curved arrow) and a hypoplastic right vertebral artery (white oblique arrow).

and basilar artery were present, and the right posterior communicating artery was feeding the anterior circulation (Figure 2f). The constellation of imaging findings confirmed the diagnosis of PHACES syndrome. Because the heart was structurally normal, surgical intervention to cover the defect was considered. At 3 months of age, there was a $50 \%$ reduction of the exophthalmos and the facial hemangioma. In addition, there was evidence of right hemihypertrophy, with a 2-cm difference in the circumference between the right and left upper and lower limbs.

\section{DISCUSSION}

PHACES is a neurocutaneous syndrome involving a spectrum of abnormalities including posterior fossa anomalies, hemangiomas, arterial abnormalities, cardiac defects, abnormalities of the eye, and sternal clefting. ${ }^{4}$ PHACES syndrome may be present in up to $2 \%$ of children with facial hemangiomas and $20 \%$ of children with segmental facial hemangiomas. ${ }^{2}$ Most of the cardiac anomalies described involve aortic arch pathology, abnormalities ranging from coarctation to aneurysm formation, atrial and ventricular septal defects, patent ductus arteriosus, persistent left superior vena cava, and abnormal brachiocephalic vessels. ${ }^{5}$ To our knowledge, only one case of complete ectopia cordis associated with PHACES syndrome has been reported in the literature. ${ }^{2}$ In addition, hemihypertrophy has never been described as associated with PHACES syndrome.

Based on the consensus guidelines published in 2016, the definite diagnosis of PHACE syndrome requires the presence of infantile hemangioma of the face or scalp $>5 \mathrm{~cm}$ in diameter and one major diagnostic criterion. In addition, patients with large segmental infantile hemangioma of the neck, upper trunk, or trunk and proximal upper extremity who also have two other major criteria should be considered to have definite PHACE. ${ }^{6}$

In our case, the partial ectopia cordis with sternal clefting was the major presenting symptom that prompted further workup. It varies from a minor " $V$ "-shaped defect to complete separation of sternal halves. In cases of complete thoracic ectopia cordis, which is an extremely rare malformation, the naked heart is displaced outside the thoracic cavity without pericardial coverage. ${ }^{2,3}$ However, in partial ectopia cordis, the heart is often seen to pulsate and is covered with a thin layer of skin. $^{2}$ Superior sternal cleft is the most common variant associated with PHACES syndrome. ${ }^{2}$ On plain chest radiography, sternal cleft could be suggested by widely spaced anterior ribs and the proximal ends of the clavicles. However, further clarification can be achieved by computed tomography. ${ }^{7}$

The association of hemihypertrophy with PHACES syndrome has not been previously reported. Hemihypertrophy, more accurately referred to as hemihyperplasia, is a rare overgrowth disorder of unknown etiology that is characterized by enlargement of part or all of one side of the body. It can be sporadic or in association with other syndromes like Beckwith-Wiedemann syndrome, Proteus syndrome, Klippel-Trenaunay syndrome, Russell-Silver syndrome, and neurofibromatosis type $1 .^{8}$ Several reports showed an increased risk of neoplasms in overgrowth disorders, in particular embryonal tumors, which warrants close follow-up with serum markers and imaging. ${ }^{9,10}$ Likewise, ectopia cordis 
can be seen either in isolation or in association with syndromes, such as the pentalogy of Cantrell. ${ }^{11}$ Those can be differentiated by clinical criteria and imaging findings.

Once the diagnosis of PHACES syndrome is suspected, a complete physical examination should be performed. Radiologists must also be aware of various imaging features of PHACE(S) syndrome. ${ }^{4}$ In particular, look for specific arterial anomalies of the head and neck, including cerebral aneurysm, stenosis, aberrant origin or course of the ICA, hypoplasia or agenesis of the ICA or vertebral arteries, and persistent primitive vertebrobasilar anastomosis. Posterior fossa abnormalities range from a classic Dandy-Walker malformation to ipsilateral cerebellar hypoplasia, cerebellar vermian hypoplasia, and cortical dysgenesis, as well as arachnoid cysts. ${ }^{12}$ Other anomalies include hypoplasia or agenesis of the corpus callosum and intracranial hemangiomas. ${ }^{13}$ The presence of intracranial vascular anomalies significantly increases the risk of arterial ischemic stroke in children with $\operatorname{PHACE}(S) .{ }^{14}$ Therefore, MRI with gadolinium and MRA of the head should also be performed when PHACES is suspected.

\section{ORCID}

Jad Chokr (iD http://orcid.org/0000-0002-2478-9195

Bedros Taslakian (iD http://orcid.org/0000-0002-7542-089X

Gilbert Maroun (D) http://orcid.org/0000-0001-7045-1534

Gagandeep Choudhary (D) http://orcid.org/0000-0002-3261-1979

1. Sullivan C, Christian S, Shieh J, et al. X chromosome-inactivation patterns in 31 individuals with PHACE syndrome. Mol Syndromol 2013;4(3):114-118.

2. Lopez-Gutierrez JC. PHACES syndrome and ectopia cordis. Interact Cardiovasc Thorac Surg. 2011;12(4):642-644. doi:10.1510/ icvts.2010.258442.
3. Engum SA. Embryology, sternal clefts, ectopia cordis, and Cantrell's pentalogy. Semin Pediatr Surg. 2008;17(3):154-160. doi:10.1053/ j.sempedsurg.2008.03.004.

4. Merheb M, Hourani R, Zantout M, Azar S. Endocrine dysfunction in a patient with PHACE syndrome, including port-wine stain of the right periorbital area. Endocr Pract. 2010;16(2):255-259. doi: 10.4158/EP09179.CR.

5. Metry DW, Garzon MC, Drolet BA, et al. PHACE syndrome: current knowledge, future directions. Pediatr Dermatol. 2009;26(4): 381-398. doi:10.1111/j.1525-1470.2009.00944.x.

6. Garzon MC, Epstein LG, Heyer GL, et al. PHACE syndrome: consensus-derived diagnosis and care recommendations. J Pediatr. 2016; 178:24-33. doi:10.1016/j.jpeds.2016.07.054

7. Restrepo CS, Martinez S, Lemos DF, et al. Imaging appearances of the sternum and sternoclavicular joints. Radiographics. 2009;29(3): 839-859. doi:10.1148/rg.293055136.

8. Clericuzio CL, Martin RA. Diagnostic criteria and tumor screening for individuals with isolated hemihyperplasia. Genet Med. 2009;11(3): 220-222. doi:10.1097/GIM.0b013e31819436cf.

9. Clericuzio CL, Chen E, McNeil DE, et al. Serum alpha-fetoprotein screening for hepatoblastoma in children with Beckwith-Wiedemann syndrome or isolated hemihyperplasia. J Pediatr. 2003;143(2): 270-272. doi:10.1067/S0022-3476(03)00306-8.

10. Hoyme HE, Seaver LH, Jones KL, Procopio F, Crooks W, Feingold M. Isolated hemihyperplasia (hemihypertrophy): report of a prospective multicenter study of the incidence of neoplasia and review. $A m \mathrm{~J}$ Med Genet. 1998;79(4):274-278.

11. Kumar B, Sharma C, Sinha DD. Ectopia cordis associated with Cantrell's pentalogy. Ann Thorac Med. 2008;3(4):152-153.

12. Pascual-Castroviejo I. Vascular and nonvascular intracranial malformations associated with external capillary hemangiomas. Neuroradiologicum. 1978; 16:82-84.

13. Judd C, Chapman P, Koch B, Shea C. Intracranial infantile hemangiomas associated with PHACE syndrome. Am J Neuroradiol. 2007; 28(1):25-29.

14. Siegel DH, Tefft KA, Kelly T, et al. Stroke in children with posterior fossa brain malformations, hemangiomas, arterial anomalies, coarctation of the aorta and cardiac defects, and eye abnormalities (PHACE) syndrome: a systematic review of the literature. Stroke. 2012;43(6): 1672-1674. doi:10.1161/STROKEAHA.112.650952. 